\title{
Area, Curve Complexity, and Crossing Resolution of Non-planar Graph Drawings
}

\author{
Emilio Di Giacomo ${ }^{1}$, Walter Didimo ${ }^{1}$, Giuseppe Liotta ${ }^{1}$, and Henk Meijer ${ }^{2}$ \\ ${ }^{1}$ Dip. di Ingegneria Elettronica e dell’Informazione, Università degli Studi di Perugia \\ \{digiacomo, didimo, liotta\}@diei.unipg.it \\ ${ }^{2}$ Roosevelt Academy, The Netherlands \\ h.meijer@roac.nl
}

\begin{abstract}
In this paper we study non-planar drawings of graphs, and study tradeoffs between the crossing resolution (i.e., the minimum angle formed by two crossing segments), the curve complexity (i.e., maximum number of bends per edge), the total number of bends, and the area.
\end{abstract}

\section{Introduction}

Planarity is one of the most desirable properties when drawing a graph because planar drawings are more readable and more aesthetically pleasant than non-planar ones. Unfortunately, very few graphs are planar in practice and edge crossings are unavoidable in the vast majority of the application scenarios where relational data are visualized and analyzed by means of graph drawing techniques.

While a large body of literature has been published about the problem of reducing the number of crossings in a non-planar drawing of a graph, by the well-known crossinglemma this number is quadratic with the number of the edges for dense graphs. This, together with cognitive experiments showing the negative impact of edge crossings on the human understanding of a graph drawing, apparently leads to the conclusion that computing readable node-link visualizations of dense graphs is a hopeless challenge [67/8].

However, new cognitive experiments on large drawings of graphs refine the conclusions in 6 678 by showing that actually the human's understanding of the relations between the nodes of a network is not bothered by those edge crossings that form large angles [3|4|5]. These experiments suggest a new and fascinating research scenario in which the problem of maximizing the crossing resolution of a drawing (i.e. the smallest angle formed by two crossing edges) becomes as important for the non-planar graphs as the problem of avoiding edge crossings is for the planar graphs.

With this motivation, we study the trade-offs between crossing resolution, curve complexity, total number of bends, and area of non-planar drawings of graphs. The area is measured as the number of grid points contained in or on a bounding box of the drawing, i.e., the smallest axis-aligned box enclosing the drawing. The curve complexity is the maximum number of bends along each edge of the drawing.

We recall that the special case where the crossing resolution of a drawing is $\frac{\pi}{2}$ has been studied in [1], where these types of drawings are called Right Angle Crossing drawings ( $R A C$ drawings, for short). The main theme of [1] is to study the trade-off

D. Eppstein and E.R. Gansner (Eds.): GD 2009, LNCS 5849, pp. 15-20, 2010.

(C) Springer-Verlag Berlin Heidelberg 2010 
between the edge density and the curve complexity in a $R A C$ drawing. In this paper we extend the study of [1] in two ways: we take into account the area requirement and we relax the crossing resolution constraints to angles smaller than $\frac{\pi}{2}$. Our main results can be listed as follows.

- We study the trade-off between area requirement and curve complexity of $R A C$ drawings. We establish an $\Omega\left(n^{2}\right)$ lower bound on the area requirement of $R A C$ drawings and show an $O(n+m)$-time algorithm whose input is a graph $G$ with $n$ vertices and $m$ edges and whose output is a $R A C$ drawing of $G$ having curve complexity 4, total number of bends $O(m)$, and area $O\left(n^{3}\right)$. We observe that the previously known algorithm requires area $O\left(n^{4}\right)$ and curve complexity 3 [1].

- We relax the constraint on the crossing resolution and introduce the Large Angle Crossing drawings ( $L A C$ drawings, for short). In a $L A C$ drawing the edge crossings are allowed to form angles at least $\frac{\pi}{2}-\varepsilon$ for any given $0<\varepsilon<\frac{\pi}{2}$. For any choice of the constant $\varepsilon$, we show an infinite family of graphs such that any $L A C$ drawing of an $n$-vertex graph in the family requires curve complexity 1 , total number of bends $\Omega\left(n^{2}\right)$, and $\Omega\left(n^{2}\right)$ area.

- We describe an $O(n+m)$-time algorithm whose input is a graph $G$ with $n$ vertices and $m$ edges and a constant $0<\varepsilon<\frac{\pi}{2}$ and whose output is a $L A C$ drawing of $G$ having crossing resolution $\frac{\pi}{2}-\varepsilon$, curve complexity 1 , total number of bends $O(m)$, and area $O\left(n^{2}\right)$, which are worst-case optimal.

The rest of this paper is organized as follows. Preliminary definitions are given in Section 2. Results about $R A C$ drawings and $L A C$ drawings are presented in Section 3 and 4 respectively. Section 5 lists some open problems. Some proofs are sketched or omitted for reasons of space.

\section{Preliminaries}

Let $G$ be a graph. A polyline drawing $\Gamma$ of $G$ is a geometric representation of $G$ such that each vertex $u$ of $G$ is mapped to a distinct point $p_{u}$ of the plane, each edge $(u, v)$ of $G$ is drawn as a polyline with end-points $p_{u}$ and $p_{v}$, and two edges can intersect either at shared endvertices or at a finite number of interior points. Each intersection between two or more edges that happens at an interior point is called a crossing. Since each edge is drawn as a polyline, each crossing is an intersection of two or more straight-line segments. The intersection angle between two crossing straight-line segments is the smallest angle defined by these segments. The crossing resolution of a polyline drawing is the minimum intersection angle between any two segments. Each point shared by two consecutive segments of a polyline representing an edge is called a bend. The curve complexity of a polyline drawing is the maximum number of bends along an edge. A straight-line drawing is a polyline drawing with curve complexity zero. A polyline drawing is a grid drawing if the points representing vertices and bends have integer coordinates. The bounding box of a polyline grid drawing $\Gamma$ of a graph $G$ is the smallest axis-aligned rectangle containing $\Gamma$. If the sides of the bounding box of $\Gamma$ parallel to the $x$ - and $y$-axis have lengths $W-1$ and $H-1$, respectively, we say that $\Gamma$ has width $W$, height $H$ and area $W \cdot H$. A polyline grid drawing with crossing resolution $\frac{\pi}{2}$ is 
called a $R A C$ drawing. A polyline grid drawing with crossing resolution $0<\alpha<\frac{\pi}{2}$ is called a $L A C_{\alpha}$ drawing. We will write $L A C$-drawing when we are not interested in the value of $\alpha$.

\section{Optimal Crossing Resolution: RAC Drawings}

The following results, consequence of the results in [1], establish lower and upper bounds on the curve complexity, on the total number of bends and on the area of $R A C$ drawings of graphs.

Theorem 1. There exists an infinite family of graphs such that any $R A C$ drawing of an $n$-vertex graph in the family has curve complexity at least 3 , total number of bends $\Omega\left(n^{2}\right)$ and area $\Omega\left(n^{2}\right)$.

Lemma 1. Every graph with $n$ vertices and $m$ edges admits a $R A C$ drawing with curve complexity 3, total number of bends $O(m)$ and area $O\left(n^{4}\right)$.

We prove now that the upper bound on the area of a $R A C$ drawing can be reduced to $O\left(n^{3}\right)$ at the expense of the curve complexity that increases to 4 .

Lemma 2. The complete graph $K_{n}$ admits a RAC drawing with curve complexity 4, total number of bends $O\left(n^{2}\right)$, and area $O\left(n^{3}\right)$.

Sketch of Proof: Refer to Fig. 1 for an illustration of the technique with $n=6$. Arbitrarily number the vertices of $K_{n}$ from 0 to $n-1$. Vertex $i$ with $0 \leq i \leq n-1$ is placed at point $p_{i}=(i n-3,2 n)$. For each pair of vertices $i$ and $j$, with $i<j$, the four bends of edge $(i, j)$ will be placed at the following points (in this order): $a_{i, j}=$ $(i n-2,(j-i)-1+2(n-1)), b_{i, j}=(i n,(j-i)-1), c_{i, j}=(j n-(j-i), 2(j-i)-1)$, and $d_{i, j}=(j n-(j-i)-2,2(j-i)-1+2(n-1))$.

Clearly the drawing has curve complexity 4 and total number of bends $4 \frac{n(n-1)}{2}$. Also, it is easy to see that the crossing resolution is $\frac{\pi}{2}$. We now prove that the area is $O\left(n^{3}\right)$. The point with smallest $x$-coordinate is $p_{0}=(-3,2 n)$, while the point with largest $x$-coordinate is $c_{n-2, n-1}=\left(n^{2}-n-1,1\right)$ and therefore the width of the drawing is $n^{2}-n-1+3+1=n^{2}-n+3=O\left(n^{2}\right)$. The points with smallest $y$ coordinates are the points $b_{i, i+1}=(\mathrm{in}, 0)$, while the point with largest $y$-coordinate is $d_{0, n-1}=\left(n^{2}-2 n-1,4 n-5\right)$, and therefore the height of the drawing is $4 n-5+1=$ $4 n-4=O(n)$ which gives an $O\left(n^{3}\right)$ area.

The following theorem summarizes the results about the upper bounds

Theorem 2. Every graph with $n$ vertices and $m$ edges admits both a $R A C$ drawing with curve complexity 3, total number of bends $O(m)$, and area $O\left(n^{4}\right)$ and a $R A C$ drawing with curve complexity 4, total number of bends $O(m)$ and area $O\left(n^{3}\right)$. Both drawings can be computed in $O(n+m)$ time.

In the next section we show that a drawing that is worst-case optimal in terms of curve complexity, total number of bends, and area can be computed if one allows a crossing resolution arbitrarily close to the optimal one. 


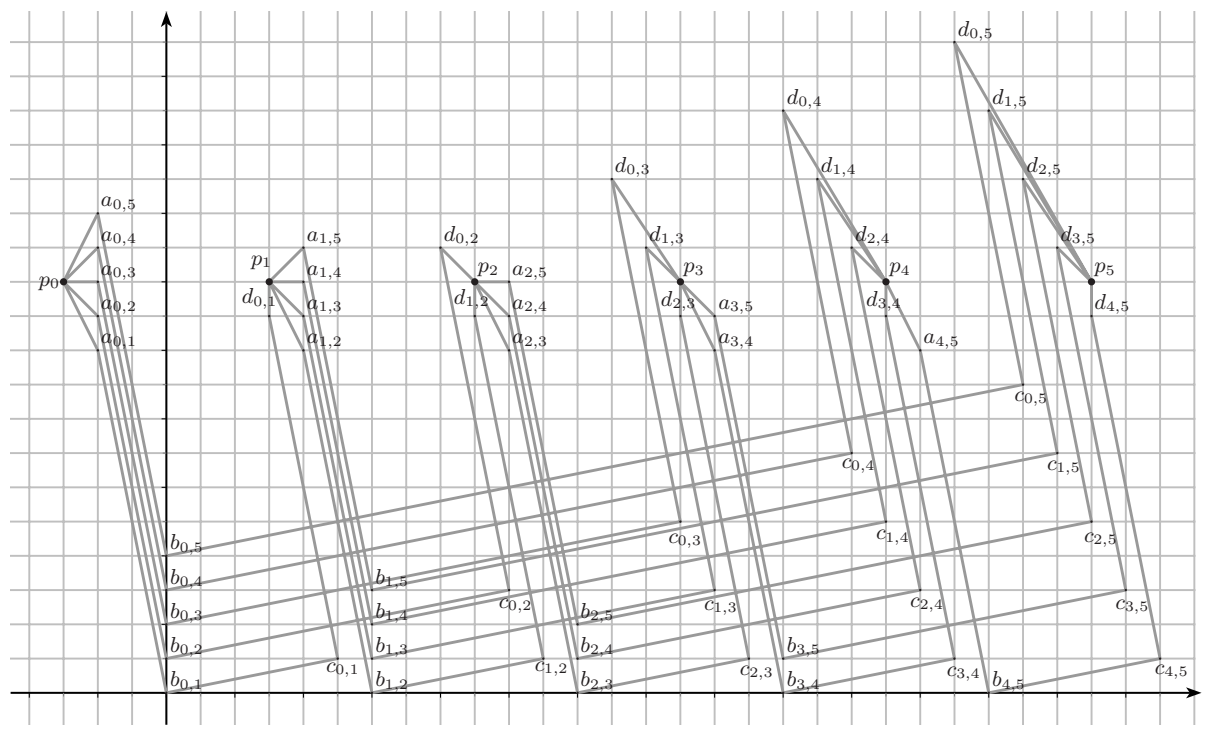

Fig. 1. A $R A C$ drawing of $K_{6}$ with curve complexity 4

\section{Nearly Optimal Crossing Resolution: LAC Drawings}

We start by proving lower bounds on the curve complexity, on the total number of bends, and on the area of $L A C_{\frac{\pi}{2}-\varepsilon}$ drawings for every $0 \leq \varepsilon \leq \frac{\pi}{2}$. The next three lemmas are the technical foundation of our lower bound. The proof of the first one is omitted for reasons of space.

Lemma 3. In a straight-line drawing of $K_{n}$ on a convex set of $n>3$ point there exists an intersection angle $\leq \frac{2 \pi}{n}$.

Lemma 4. For all $\varepsilon>0$ there exists a constant $N$ such that for all $n \geq N$, any straight line drawing of $K_{n}$ has an intersection angle $<\varepsilon$.

Proof. Let $\varepsilon>0$. Let $k>2 \pi / \varepsilon$. From the Erdös-Szekeres lemma [2] there exists an $N$ such that any set of $n \geq N$ non-degenerate points (i.e. no three points collinear) contains a set of $k$ convex points. Consider a straight-line drawing of $K_{n}$ with $n \geq N$. If there are three collinear points in this drawing, there are two edges intersecting at an infinite number of points, i.e., the drawing is not a polyline drawing according to our definition. If there are no three collinear points, then there is a subset of $k$ convex points. From Lemma 3 the drawing of $K_{n}$ contains an intersection angle $\leq \frac{2 \pi}{k}<\varepsilon$.

Lemma 5. For all $\varepsilon>0$ there exist constants $N$ and $c_{N}$ such that for all $n>N$, any subdrawing obtained removing at most $n(n-1) / c_{N}$ edges from a straight-line drawing of $K_{n}$ has an intersection angle $\leq \varepsilon$.

Proof. Let $\varepsilon>0$. From Lemma 4 we know that there is a constant $N$ such that any straight-line drawing of $K_{N}$ has an intersection angle less than $\varepsilon$. Let $c_{N}=2 N(N-1)$ 
and let $n>N$. Consider a straight-line drawing of $K_{n}$. Any edge of $K_{n}$ is in $\left(\begin{array}{c}n-2 \\ N-2\end{array}\right)$ different subgraphs of $K_{n}$ isomorph to $K_{N}$. So by removing $\leq n(n-1) / c_{N}$ edges from the drawing we remove at most $\frac{n(n-1)}{c_{N}}\left(\begin{array}{c}n-2 \\ N-2\end{array}\right)$ copies of $K_{N}$. Therefore, there are at least $\left(\begin{array}{c}n \\ N\end{array}\right)-\frac{n(n-1)}{c_{N}}\left(\begin{array}{c}n-2 \\ N-2\end{array}\right)$ copies of $K_{N}$ present in the drawing. Since $\left(\begin{array}{c}n \\ N\end{array}\right)-\frac{n(n-1)}{c_{N}}\left(\begin{array}{c}n-2 \\ N-2\end{array}\right)=$ $\left(\begin{array}{c}n \\ N\end{array}\right)-\frac{n(n-1)(n-2) !}{2 N(N-1)(N-2) !(n-N) !}=\left(\begin{array}{l}n \\ N\end{array}\right)-\frac{n !}{2 N !(n-N) !}=\frac{1}{2}\left(\begin{array}{l}n \\ N\end{array}\right)>1$, there is an intersection angle less than $\varepsilon$.

Theorem 3. For every $0<\varepsilon<\frac{\pi}{2}$ there exists a constant $N$ such that for every $n>N$ there exists a graph $G$ with $n$ vertices such that every $L A C_{\frac{\pi}{2}-\varepsilon}$ drawing of $G$ has curve complexity at least 1 , total number of bends $\Omega\left(n^{2}\right)$ and area $\Omega\left(n^{2}\right)$.

Proof. From Lemma 5 we know that for any value of $\frac{\pi}{2}-\varepsilon$ there are constants $N$ and $c_{N}$ such that for any straight-line drawing of $K_{n}$ with $n>N$, even if we remove at most $n(n-1) / c_{N}$ of the edges of $K_{n}$ there is an intersection angle less than $\frac{\pi}{2}-\varepsilon$. Thus, a drawing with intersection angles at least $\frac{\pi}{2}-\varepsilon$ has at least $n(n-1) / c_{N}$ bent edges. Since bends have to be placed on grid points, the result follows.

We prove now that the lower bounds of Theorem 3 are worst-case optimal.

Lemma 6. For every $0<\varepsilon<\frac{\pi}{2}$ and for all $n>3$ there exists a $L A C_{\frac{\pi}{2}-\varepsilon}$ drawing of $K_{n}$ with curve complexity 1 , total number of bends $O\left(n^{2}\right)$, and area $O\left(n^{2}\left(\cot \frac{\varepsilon}{2}\right)^{2}\right)$.

Sketch of Proof: Let $c$ be an integer such that the angle between a line of slope $c+1$ and a line of slope $\frac{1}{(c+1)}$ is larger than $\frac{\pi}{2}-\varepsilon$. Arbitrarily number the vertices of $K_{n}$ from 0 to $n-1$. Refer to Fig.2 for an example with $n=6$ and $c=1$. Vertex $i$ with $0 \leq i \leq n-1$ is placed at point $p_{i}=(i c,(n-i-1) c)$. For each pair of vertices $i$ and $j$, with $i<j$, the bend of edge $(i, j)$ will be placed at point $a_{i, j}=(j c+1,(n-i-1) c+1)$. Clearly, the curve complexity is 1 and the total number of bends is $\frac{n(n-1)}{2}$. It is easy to see that

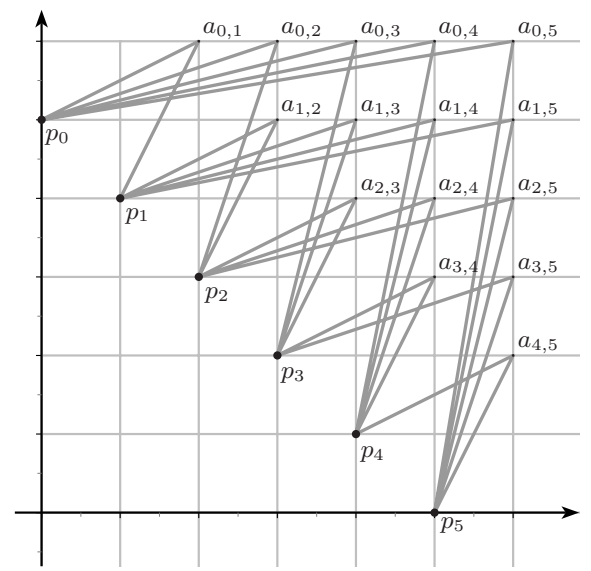

Fig. 2. A $L A C_{\alpha}$-drawing of $K_{6}$ with curve complexity $1\left(\alpha>63^{\circ}\right)$ 
the crossing resolution is larger than $\frac{\pi}{2}-\varepsilon$ and that the area is $O\left(c^{2} n^{2}\right)$. With simple geometric arguments it can be proved that $c=O\left(\cot \frac{\varepsilon}{2}\right)$.

The following theorem summarizes the results of this section.

Theorem 4. For every $0<\varepsilon<\frac{\pi}{2}$, every graph with $n$ vertices admits a $L A C_{\frac{\pi}{2}}-\varepsilon$ drawing which is worst-case optimal in terms of curve complexity, total number of bends, and area. Namely, the curve complexity is 1, the total number of bends is $\Theta(m)$, and the area is $\Theta\left(n^{2}\right)$. Furthermore, the drawing can be computed in $O(n+m)$ time.

\section{Open Problems}

We conclude by listing some open problems that arise from the results of this work. One natural problem is that of closing the gap between the upper and the lower bound on the area of $R A C$ drawings stated by Theorem 1 and Theorem 2 .

The lower bounds on the area of $R A C$ and $L A C$ drawings are a consequence of the fact that $K_{n}$ contains $O\left(n^{2}\right)$ edges. It would be interesting to study whether a $o\left(n^{2}\right)$ area and good crossing resolution can be obtained for graphs with $O(n)$ edges.

A question related to the previous one is the following: Is it possible to obtain straight-line drawings of planar graphs with $o\left(n^{2}\right)$ area if we allow right or large angle crossings? It is worth mentioning that, if no requirement about the crossing resolution exists, then every planar graph admits a non-planar drawing in $O(n)$ area [9].

\section{References}

1. Didimo, W., Eades, P., Liotta, G.: Drawing Graphs with Right Angle Crossings. In: Dehne, F., et al. (eds.) WADS 2009. LNCS, vol. 5664, pp. 206-217. Springer, Heidelberg (2009)

2. Erdös, P., Szekeres, G.: A combinatorial problem in geometry. Compositio Math. 2, 463-470 (1935)

3. Huang, W.: Using eye tracking to investigate graph layout effects. In: Hong, S.H., Ma, K.L. (eds.) APVIS, pp. 97-100. IEEE, Los Alamitos (2007)

4. Huang, W.: An eye tracking study into the effects of graph layout. CoRR abs/0810.4431 (2008)

5. Huang, W., Hong, S.H., Eades, P.: Effects of crossing angles. In: PacificVis, pp. 41-46. IEEE, Los Alamitos (2008)

6. Purchase, H.C.: Effective information visualisation: a study of graph drawing aesthetics and algorithms. Interacting with Computers 13(2), 147-162 (2000)

7. Purchase, H.C., Carrington, D.A., Allder, J.A.: Empirical evaluation of aesthetics-based graph layout. Empirical Software Engineering 7(3), 233-255 (2002)

8. Ware, C., Purchase, H.C., Colpoys, L., McGill, M.: Cognitive measurements of graph aesthetics. Information Visualization 1(2), 103-110 (2002)

9. Wood, D.R.: Grid drawings of k-colourable graphs. Computational Geometry 30(1), 25-28 (2005) 